\title{
Notes on Use of the Composite Estimator: an Improvement of the Ratio Estimator
}

\author{
Kung-Jong Lui ${ }^{1}$
}

\begin{abstract}
This article discusses use of the composite estimator with the optimal weight to reduce the variance (or the mean-squared-error, MSE) of the ratio estimator. To study the practical usefulness of the proposed composite estimator, a Monte Carlo simulation is performed comparing the bias and MSE of composite estimators (with estimated optimal weight and with known optimal weight) with those of the simple expansion and the ratio estimators. Two examples, one regarding the estimation of dead fir trees via an aerial photo and the other regarding the estimation of the average sugarcane acres per county, are included to illustrate the use of the composite estimator developed here.
\end{abstract}

Key words: Composite estimator; ratio estimator; simple expansion estimator; odds ratio; phi correlation; regression estimator.

\section{Introduction}

The ratio estimator, which incorporates the information on the auxiliary measurements into estimation, is one of the most commonly-used estimators in surveys. For example, the Current Population Survey employed the ratio estimator accounting for the number of African Americans to estimate the number of unemployed African Americans (Scheaffer et al. 2012). When the correlation between the auxiliary measurement and the studied measurement is high, the ratio estimator can outperform the simple expansion estimator with respect to precision (or variance) (Cochran 1977, 157-158). However, if this correlation is low, the ratio estimator can be less precise than the simple expansion estimator (Cochran 1977). The ratio estimator is biased, but its bias decreases to 0 as the sample size increases to $\infty$ (Cochran 1977). There are publications on adjusting or reducing the bias of the ratio estimator (Pascual 1961; Sahoo 1987; Cochran 1977). Because the bias of the ratio estimator is of order $1 / n$, we commonly focus our attention on variance when comparing the ratio estimator with the simple expansion estimator (Cochran 1977; Scheaffer et al. 2012). Some discussions on use of the composite estimation technique to improve the precision of existing estimators in small area estimation (Lui and Cumberland 1991; Schaible 1978, Royall 1970) or to extend the ratio estimator to multivariate ratio-typo estimators have been presented elsewhere (Sukhatme et al. 1984, 217-223; Cochran 1977, 184-185). A recent discussion on use of the

\footnotetext{
${ }^{1}$ Department of Mathematics and Statistics, San Diego State University, San Diego, CA 92182-7720. USA. Email: klui@sdsu.edu

Acknowledgments: The author wishes to thank the Associate Editor and four Referees for many useful and valuable comments to improve the contents and clarity of this article.
} 
composite estimator with weights proportional to the reciprocal of the variance of its component estimator for two-stage cluster sampling has also been presented by Lee et al. (2016). However, the weight suggested by Lee et al. (2016) is optimal only when its two component estimators are independent.

This article suggests that the idea of the composite estimator to reduce the bias and variance (or the mean-squared-error (MSE) of the ratio estimator may be applied with no additional efforts of collecting extra data. The optimal weight minimizing variance (or MSE) for the composite estimator under the simple random sampling (SRS) is derived. A Monte Carlo simulation comparing the performance of the simple expansion estimator, the ratio estimator, the composite estimator with an estimated optimal weight and the composite estimator with known optimal weight is carried out in a variety of situations. A brief discussion is given on deriving the optimal weight minimizing variance (or MSE) when measurements are dichotomous. Two examples, one regarding the estimation of dead fir trees via an aerial photo and the other regarding the estimation of the average sugarcane acres per county, are included to illustrate the use of the composite estimator developed here.

\section{Notation and Methods}

Suppose that a population consists of $\left\{\left(X_{i}, Y_{i}\right) \mid X_{i}>0, Y_{i}>0, i=1,2,3, \ldots, N\right\}$, where $X_{i}$ and $Y_{i}$ are measurements on subject $i$ and $N$ is the population size. For clarity, we summarize the definitions of notation in the following:

Let $X=\sum_{i=1}^{N} X_{i}$ and $Y=\sum_{i=1}^{N} Y_{i}$ denote the population totals of measurements $X_{i}$ and $Y_{i}$; let $\bar{X}=\sum_{i=1}^{N} X_{i} / N$ and $\bar{Y}=\sum_{i=1}^{N} Y_{i} / N$ denote the population means of measurements $X_{i}$ and $Y_{i}$;

let $R=Y / X=\bar{Y} / \bar{X}$ denote the population total or mean ratio;

let $S_{x}^{2}=\sum_{i=1}^{N}\left(X_{i}-\bar{X}\right)^{2} /(N-1)$ and $S_{y}^{2}=\sum_{i=1}^{N}\left(Y_{i}-\bar{Y}\right)^{2} /(N-1)$ denote the population variances;

let $S_{x y}=\sum_{i=1}^{N}\left(X_{i}-\bar{X}\right)\left(Y_{i}-\bar{Y}\right) /(N-1)$ denote the population covariance between $X_{i}$ and $Y_{i}$;

let $\rho=S_{x y} /\left(S_{x} S_{y}\right)$ denote the simple correlation coefficient between $X_{i}$ and $Y_{i}$, as well as let $C V_{x}=S_{x} / \bar{X}$ and $C V_{y}=S_{y} / \bar{Y}$ denote the coefficients of variation for $X_{i}$ and $Y_{i}$.

Note that since we will consider using the ratio estimator only when $X_{i}$ and $Y_{i}$ are positively correlated, we assume $\rho>0$ in the following discussion.

Suppose that we employ the SRS scheme and obtain $n$ subjects. Let notation $c$ denote the collection of labels in the sample. Furthermore,

let $\bar{x}_{s}=\sum_{i \in c} X_{i} / n$ and $\bar{y}_{s}=\sum_{i \in c} Y_{i} / n$ denote the sample means of $X_{i}$ and $Y_{i}$;

let $s_{x}^{2}=\sum_{i \in c}\left(X_{i}-\bar{x}_{s}\right)^{2} /(n-1)$ and $s_{y}^{2}=\sum_{i \in c}\left(Y_{i}-\bar{y}_{s}\right)^{2} /(n-1)$ denote the sample variances;

let $\hat{R}=\bar{y}_{s} / \bar{x}_{s}$ denote the ratio of the two sample means;

let $s_{x y}=\sum_{i \in c}\left(X_{i}-\bar{x}_{s}\right)\left(Y_{i}-\bar{y}_{s}\right) /(n-1)$ denote the sample covariance between $X_{i}$ and $Y_{i}$; let $\hat{\rho}=s_{x y} /\left(s_{x} s_{y}\right)$ denote the estimated correlation coefficient between $X_{i}$ and $Y_{i}$; as well as let $c v_{x}=s_{x} / \bar{x}_{s}$ and $c v_{y}=s_{y} / \bar{y}_{s}$ denote the sample coefficients of variation for $X_{i}$ and $Y_{i}$. 
Here, we focus our attention on estimation of the population total $Y$. Note that the simplest unbiased estimator for $Y$ under the SRS is the simple expansion estimator given by (Cochran 1977)

$$
\hat{Y}=N \bar{y}_{s}
$$

The variance for $\hat{Y}$ is (Cochran 1977, 23)

$$
\begin{aligned}
\operatorname{Var}(\hat{Y}) & =N^{2}(1-f) S_{y}^{2} / n \\
& =N^{2}(1-f) \bar{Y}^{2} C V_{y}^{2} / n,
\end{aligned}
$$

where $f=n / N$ is the sampling fraction. To estimate the population total $Y$, we can also employ the most commonly-used ratio estimator defined by (Cochran 1977)

$$
\hat{Y}_{R}=\hat{R} X
$$

where $\hat{R}=\bar{y}_{s} / \bar{x}_{s}$. The variance for $\hat{Y}_{R}$ is approximately given by (Cochran 1977,154 ; see also Appendix 1, Subsection 7.1.)

$$
\begin{aligned}
\operatorname{Var}\left(\hat{Y}_{R}\right) & =N^{2}(1-f)\left[S_{y}^{2}+R^{2} S_{x}^{2}-2 R S_{X Y}\right] / n \\
& =N^{2}(1-f) \bar{Y}^{2}\left[C V_{y}^{2}+C V_{x}^{2}-2 \rho C V_{x} C V_{y}\right] / n
\end{aligned}
$$

where $R=\bar{Y} / \bar{X}$. Furthermore, we can show that the covariance between $\hat{Y}$ and $\hat{Y}_{R}$ can be approximated by (Appendix 1)

$$
\operatorname{Cov}\left(\hat{Y}, \hat{Y}_{R}\right)=N^{2}(1-f) \bar{Y}^{2}\left[C V_{y}^{2}-\rho C V_{x} C V_{y}\right] / n .
$$

Consider use of the composite estimator $w \hat{Y}+(1-w) \hat{Y}_{R}$ (where $\left.0 \leq w \leq 1\right)$ to estimate the population total $Y$. Note that the simple expansion estimator $\hat{Y}(1)$, the ratio estimator $\hat{Y}_{R}$ (3) and the composite estimator $w \hat{Y}+(1-w) \hat{Y}_{R}$ can all be expressed as $\sum_{i \in c} a_{i} y_{i}$, a linear combination of the sampled units. The weights $a_{i}$ for $\hat{Y}_{R}$ are calibrated towards the population total $X$ (i.e., $\sum_{i \in c} a_{i} x_{i}=X$ ), while the weights $a_{i}$ for $\hat{Y}$ and $w \hat{Y}+(1-w) \hat{Y}_{R}$ are not. Note also that since the weight $w$ in $w \hat{Y}+(1-w) \hat{Y}_{R}$ is between 0 and 1, the absolute magnitude of the bias for the composite estimator $\left|E\left(w \hat{Y}+(1-w) \hat{Y}_{R}-Y\right)\right|=\left|(1-w) E\left(\hat{Y}_{R}-Y\right)\right|$, is always smaller than $\left|E\left(\hat{Y}_{R}-Y\right)\right|$, the absolute magnitude of the bias for the ratio estimator. Note that the ratio estimator is an asymptotically unbiased estimator for $Y$ (Cochran 1977, 161) and so is the composite estimator. The optimal weight $w$ minimizing the $\operatorname{variance} \operatorname{Var}\left(w \hat{Y}+(1-w) \hat{Y}_{R}\right)$ can be shown to equal

$$
\begin{aligned}
w^{*} & =\left(\operatorname{Var}\left(\hat{Y}_{R}\right)-\operatorname{Cov}\left(\hat{Y}, \hat{Y}_{R}\right)\right) /\left(\operatorname{Var}\left(\hat{Y}_{R}\right)+\operatorname{Var}(\hat{Y})-2 \operatorname{Cov}\left(\hat{Y}, \hat{Y}_{R}\right)\right) \\
& =1-\rho C V_{y} / C V_{x}
\end{aligned}
$$

Define $w^{*}$ to be 0 if $1-\rho C V_{y} / C V_{x}<0$. Because $E\left(\hat{Y}_{R}-Y\right)^{2} \approx \operatorname{Var}\left(\hat{Y}_{R}\right)$ and $\operatorname{Cov}\left(\hat{Y}, \hat{Y}_{R}\right)=E(\hat{Y}-Y)\left(\hat{Y}_{R}-Y\right)$, the optimal weight $w^{*}$ (6) minimizing the variance of the composite estimator is approximately equivalent to minimizing its MSE. We denote the composite estimator $w^{*} \hat{Y}+\left(1-w^{*}\right) \hat{Y}_{R}$ with the optimal weight $w^{*}$ by $\hat{Y}_{C}\left(w^{*}\right)$. When $0<w^{*}<1$, the variance for $\hat{Y}_{C}\left(w^{*}\right)$ is obtained by simply substituting $w^{*}$ for $w$ in 
$\operatorname{Var}\left(\hat{Y}_{C}(w)\right)$ and is given by (Cochran 1977, 185)

$$
\begin{aligned}
\operatorname{Var}\left(\hat{Y}_{C}\left(w^{*}\right)\right) & =\left(\operatorname{Var}(\hat{Y}) \operatorname{Var}\left(\hat{Y}_{R}\right)-\left(\operatorname{Cov}\left(\hat{Y}, \hat{Y}_{R}\right)\right)^{2}\right) /\left(\operatorname{Var}(\hat{Y})+\operatorname{Var}\left(\hat{Y}_{R}\right)-2 \operatorname{Cov}\left(\hat{Y}, \hat{Y}_{R}\right)\right) \\
& =N^{2}(1-f) \bar{Y}^{2} C V_{y}^{2}\left(1-\rho^{2}\right) / n
\end{aligned}
$$

On the basis of (2) and (7), we can show that the proportional reduction of variance (PRV) by use of $\hat{Y}_{C}\left(w^{*}\right)$ instead of $\hat{Y}$ is given by

$$
P R V_{S}=\left[\operatorname{Var}(\hat{Y})-\operatorname{Var}\left(\hat{Y}_{C}\left(w^{*}\right)\right)\right] / \operatorname{Var}(\hat{Y})=\rho^{2} .
$$

Furthermore, the PRV by use of $\hat{Y}_{C}\left(w^{*}\right)$ instead of $\hat{Y}_{R}$ is given by

$$
\begin{aligned}
P R V_{R} & =\left[\operatorname{Var}\left(\hat{Y}_{R}\right)-\operatorname{Var}\left(\hat{Y}_{C}\left(w^{*}\right)\right)\right] / \operatorname{Var}\left(\hat{Y}_{R}\right) \\
& =1 /\left[1+\left(1-\rho^{2}\right) /\left(C V_{x} / C V_{y}-\rho\right)^{2}\right],
\end{aligned}
$$

which depends only on the ratio $C V_{x} / C V_{y}$ and $\rho$.

On the basis of $\operatorname{Var}(\hat{Y})$ and $\operatorname{Var}\left(\hat{Y}_{R}\right)$, we can see that $\operatorname{Var}\left(\hat{Y}_{R}\right)<\operatorname{Var}(\hat{Y})$ if and only if $\rho>C V_{x} /\left(2 C V_{y}\right)$. As compared with the definitions of (8) and (9), we can further show that $P R V_{R}<P R V_{s}$ if and only if $\operatorname{Var}\left(\hat{Y}_{R}\right)<\operatorname{Var}(\hat{Y})$ and hence $\rho>C V_{x} /\left(2 C V_{y}\right)$. In fact, we can also prove this result by directly comparing Equation (8) with Equation (9). When $\rho=C V_{x} /\left(2 C V_{y}\right)$, the two variances $\operatorname{Var}\left(\hat{Y}_{R}\right)=\operatorname{Var}(\hat{Y})$. In this case, the optimal weight $w^{*}$ becomes 0.5 and both $P R V_{S}$ and $P R V_{R}$ reduce to $C V_{x}^{2} /\left(4 C V_{y}^{2}\right)$. When $\rho=$ $C V_{x} /\left(2 C V_{y}\right)$ and $C V_{x}=2 C V_{y}, P R V_{S}$ and $P R V_{R}$ will equal 1 in this extreme case. On the other hand, if $\rho=C V_{x} / C V_{y}, P R V_{R}$ will reduce to 0 . This is because the composite estimator $\hat{Y}_{C}\left(w^{*}\right)$ with $w^{*}=0$ will be the same as the ratio estimator $\hat{Y}_{R}$. This accounts for the reason why $P R V_{R}$ is 0 .

To estimate $\operatorname{Var}(\hat{Y}), \operatorname{Var}\left(\hat{Y}_{R}\right)$ and $\operatorname{Cov}\left(\hat{Y}, \hat{Y}_{R}\right)$, we can substitute $\hat{\rho}, c v_{x}, c v_{y}$ and $\bar{y}_{s}$ for $\rho$, $C V_{x}, C V_{y}$ and $\bar{Y}$, respectively. Furthermore, to estimate the optimal weight $w^{*}$, we can similarly substitute estimators $\hat{\rho}, c v_{x}$ and $c v_{y}$ for $\rho, C V_{x}$ and $C V_{y}$, and obtain $\hat{w}^{*}$.

Note that if we want to employ the composite estimator $w \hat{\bar{Y}}+(1-w) \hat{\bar{Y}}_{R}$ (where $\hat{\bar{Y}}=\bar{y}_{s}$ and $\hat{\bar{Y}}_{R}=\hat{R} \bar{X}$ ) to estimate the population mean $\bar{Y}$, the optimal weight $w^{*}$ for the composite estimator minimizing variance $\operatorname{Var}\left(w \hat{\bar{Y}}+(1-w) \hat{\bar{Y}}_{R}\right)$ will remain the same as given in (6). We denote the composite estimator $w^{*} \hat{\bar{Y}}+\left(1-w^{*}\right) \hat{\bar{Y}}_{R}$ with $w^{*}$ by $\hat{\bar{Y}}_{C}\left(w^{*}\right)$. The variances $\operatorname{Var}(\hat{\bar{Y}}), \operatorname{Var}\left(\hat{\bar{Y}}_{R}\right)$ and $\operatorname{Var}\left(\hat{\bar{Y}}_{C}\left(w^{*}\right)\right)$ are simply equal to $\operatorname{Var}(\hat{Y}) / N^{2}, \operatorname{Var}\left(\hat{Y}_{R}\right) / N^{2}$ and $\operatorname{Var}\left(\hat{Y}_{C}\left(w^{*}\right)\right) / N^{2}$, respectively.

\section{Monte Carlo Simulations}

Because the true optimal weight $w^{*}=1-\rho C V_{y} / C V_{x}$ depends on unknown parameters, we need to employ the estimator $\hat{w}^{*}=1-\hat{\rho} c v_{y} / c v_{x}$ calculated from data in use of the composite estimator in practice. This may inflate variance (or MSE) of the composite estimator $\hat{Y}_{C}\left(w^{*}\right)$ with known optimal weight. We employ Monte Carlo simulation to compare the performance of $\hat{Y}, \hat{Y}_{R}, \hat{Y}_{C}\left(\hat{w}^{*}\right)$ and $\hat{Y}_{C}\left(w^{*}\right)$ with respect to the bias and MSE. For specified values of $S_{x}^{2}, S_{y}^{2}$ and $\rho S_{x} S_{y}$, we first generate $N=10000$ pairs of units $\left(X_{i}, Y_{i}\right)$ : $X_{i}=S_{x} Z_{1 i}+\bar{X}$ and $Y_{i}=\rho S_{y} Z_{1 i}+S_{y} \sqrt{1-\rho^{2}} Z_{2 i}+\bar{Y}$, where $Z_{1 i}$ and $Z_{2 i}$ are all mutually independent and follow the standard normal distribution with mean 0 and variance 1 . 
We arbitrarily choose $\bar{X}=\bar{Y}=10$ in the simulations. To assure that the occurrence of obtaining a simulated sample for which $X_{i}<0$ or $Y_{i}<0$ is rare, we focus our attention on $C V_{x}$ and $C V_{y} \leq 1 / 2$. We consider the situations in which the coefficients of variation $C V_{x}=0.33,0.5$ and $C V_{y}=0.25,0.33$ the correlation between $X_{i}$ and $Y_{i}, \rho=0.60,0.80$; and the sample size $n=10,25,50$. These cover the situations in which the optimal weight $w^{*}$ ranges from 0.20 to 0.70 . For each configuration determined by a combination of the above parameters, we generate a population of $N=10000$ pairs of units $\left(X_{i}, Y_{i}\right)$ via the standard normal random number generator in SAS (2009) according to the procedure as described in the above. We then take 10,000 simple random samples, each being of size $n$, from the population in calculation of the bias and MSE for $\hat{Y}, \hat{Y}_{R}, \hat{Y}_{C}\left(\hat{w}^{*}\right)$ and $\hat{Y}_{C}\left(w^{*}\right)$. We calculate the simulated bias (or MSE) for an estimator $e(Y)$ of $Y$ as the average of $(e(Y)-Y)$ (or the average of $(e(Y)-Y)^{2}$ ) over 10,000 simulated samples of size $n$. If we obtained $\hat{w}^{*}>1$ in a simulated sample, we would set $\hat{w}^{*}=1$. Similarly, if we obtained $\hat{w}^{*}<0$, we would set $\hat{w}^{*}=0$. To help readers easily see the relative performance of different estimators, we present the simulated MSE for $\hat{Y}_{R}, \hat{Y}_{C}\left(\hat{w}^{*}\right)$ and $\hat{Y}_{C}\left(w^{*}\right)$ relative to the MSE of the simple expansion estimator $\hat{Y}$. Note that because the bias for $\hat{Y}$ is theoretically zero, the simulated bias of an estimator $e(Y)$ relative to the simulated bias of $\hat{Y}$ can be potentially misleading. Thus, we present the bias itself for an estimator $e(Y)$. To help readers easily see where the bias of an estimator is non-negligible, we indicate the entry by a superscript " $\dagger$ " whenever its value is larger than 1,000 (or 1\% of the expected population total 100,000 in simulations).

To study the usefulness of $P R V_{S}$ (8) and $P R V_{R}$ (9) in practice, we compare the underlying values for these parameters with those obtained by use of $\hat{Y}_{C}\left(\hat{w}^{*}\right)$ in the same set of configurations considered previously. We calculate the simulated $P R V_{S}$ and $P R V_{R}$ as

$$
\operatorname{SPRV} V_{S}=\left[\operatorname{SMSE}(\hat{Y})-\left\{\operatorname{SMSE}\left(\hat{Y}_{C}\left(\hat{w}^{*}\right)\right)-\left(\operatorname{SBIAS}\left(\hat{Y}_{C}\left(\hat{w}^{*}\right)\right)\right)^{2}\right\}\right] / \operatorname{SMSE}(\hat{Y}),
$$

and

$$
\begin{aligned}
\operatorname{SPRV} V_{R}= & {\left[\left\{\operatorname{SMSE}\left(\hat{Y}_{R}\right)-\left(\operatorname{SBIAS}\left(\hat{Y}_{R}\right)\right)^{2}\right\}-\left\{\operatorname{SMSE}\left(\hat{Y}_{C}\left(\hat{w}^{*}\right)\right)-\left(\operatorname{SBIAS}\left(\hat{Y}_{C}\left(\hat{w}^{*}\right)\right)\right)^{2}\right\}\right] } \\
& /\left[\operatorname{SMSEE}\left(\hat{Y}_{R}\right)-\left(\operatorname{SBIAS}\left(\hat{Y}_{R}\right)\right)^{2}\right],
\end{aligned}
$$

where $\operatorname{SMSE}(\hat{Y}), \operatorname{SMSE}\left(\hat{Y}_{R}\right)$ and $\operatorname{SMSE}\left(\hat{Y}_{C}\left(\hat{w}^{*}\right)\right)$ are the simulated MSEs for $\hat{Y}, \hat{Y}_{R}$ and $\hat{Y}_{C}\left(\hat{w}^{*}\right)$, as well as $\operatorname{SBIAS}\left(\hat{Y}_{R}\right)$ and $\operatorname{SBIAS}\left(\hat{Y}_{C}\left(\hat{w}^{*}\right)\right)$ are the simulated biases for $\hat{Y}_{R}$ and $\hat{Y}_{C}\left(\hat{w}^{*}\right)$, respectively.

\section{Results}

Table 1 summarizes the simulated bias and the simulated MSE relative to the MSE of $\hat{Y}$ for estimators $\hat{Y} \hat{Y}_{R}, \hat{Y}_{C}\left(\hat{w}^{*}\right)$ and $\hat{Y}_{C}\left(w^{*}\right)$ in situations in which the coefficients of variation $C V_{x}=0.33,0.5$ and $C V_{y}=0.25,0.33$ the correlation between $X_{i}$ and $Y_{i}, \rho=0.60,0.80$ and the sample size $n=10,25,50$. As what we expect, the bias of the ratio estimator can be non-negligible, especially when the sample size $n$ is not large $(=10)$. The biases for the other estimators are generally smaller than that of the ratio estimator (Table 1). When we examine the relative simulated MSE, the composite estimator $\hat{Y}_{C}\left(w^{*}\right)$ with known optimal weight has the smallest MSE (in boldface). Table 1 also suggests that the composite estimator $\hat{Y}_{C}\left(\hat{w}^{*}\right)$ can consistently outperform the ratio estimator $\hat{Y}_{R}$ with 
Table 1. The simulated bias and the simulated MSE (in parenthesis) relative to the MSE of the simple expansion estimator $\hat{Y}$, for the ratio estimator $\hat{Y}_{R}$, the composite estimator $\hat{Y}_{C}\left(\hat{w}^{*}\right)$ with the estimated optimal weight and the composite estimator $\hat{Y}_{C}\left(w^{*}\right)$ with the known optimal weight in situations in which the coefficient of variation $C V_{x}=1 / 3,1 / 2 ; C V_{y}=1 / 4,1 / 3$; the correlation between $X_{i}$ and $Y_{i}, \rho=0.60,0.80$; and the sample size $n=10$, 25,50 . The entry with the bias larger than $1 \%$ of the expected population total is indicated by a superscript " $\dagger$ " and the entry with the smallest relative simulated MSE is printed in boldface. Each entry is calculated on the basis of 10,000 repeated samples.

\begin{tabular}{|c|c|c|c|c|c|c|c|}
\hline$C V_{x}$ & $C V_{y}$ & $\rho$ & $n$ & $\hat{Y}$ & $\hat{Y}_{R}$ & $\hat{Y}_{C}\left(\hat{w}^{*}\right)$ & $\hat{Y}_{C}\left(w^{*}\right)$ \\
\hline \multirow[t]{23}{*}{0.33} & \multirow[t]{11}{*}{0.25} & \multirow[t]{5}{*}{0.60} & 10 & $\begin{array}{c}55 \\
(1000)\end{array}$ & $\begin{array}{r}770 \\
(1289)\end{array}$ & $\begin{array}{r}66 \\
(0731)\end{array}$ & 372 \\
\hline & & & 25 & -66 & 279 & -23 & 87 \\
\hline & & & & $(1.000)$ & $(1.202)$ & $(0.667)$ & $(0.637)$ \\
\hline & & & 50 & -34 & 149 & -11 & 47 \\
\hline & & & & (1.000) & (1.221) & $(0.661)$ & $(0.651)$ \\
\hline & & \multirow[t]{6}{*}{0.80} & 10 & 1 & 468 & 32 & 281 \\
\hline & & & & $(1.000)$ & $(0.685)$ & $(0.403)$ & $(\mathbf{0 . 3 6 3})$ \\
\hline & & & 25 & -57 & 150 & -32 & 67 \\
\hline & & & & (1.000) & $(0.640)$ & $(0.354)$ & $(0.342)$ \\
\hline & & & 50 & -64 & 105 & -15 & 37 \\
\hline & & & & (1.000) & $(0.647)$ & $(0.358)$ & $(\mathbf{0 . 3 5 3})$ \\
\hline & \multirow[t]{12}{*}{0.33} & \multirow[t]{6}{*}{0.60} & 10 & 67 & 491 & 23 & 321 \\
\hline & & & & (1.000) & $(0.836)$ & (0.707) & $(\mathbf{0 . 6 5 3})$ \\
\hline & & & 25 & 23 & 173 & 15 & 113 \\
\hline & & & & (1.000) & $(0.804)$ & $(0.661)$ & $(0.640)$ \\
\hline & & & 50 & -42 & 66 & -41 & 23 \\
\hline & & & & (1.000) & $(0.784)$ & $(0.640)$ & $(\mathbf{0 . 6 3 1})$ \\
\hline & & \multirow[t]{6}{*}{0.80} & 10 & -107 & 115 & -43 & 71 \\
\hline & & & & (1.000) & $(0.404)$ & (0.391) & $(0.360)$ \\
\hline & & & 25 & 84 & 108 & 47 & 103 \\
\hline & & & & (1.000) & $(0.402)$ & (0.371) & $(0.361)$ \\
\hline & & & 50 & 59 & 24 & 1 & 31 \\
\hline & & & & $(1.000)$ & (0.393) & (0.359) & $(0.352)$ \\
\hline \multirow[t]{24}{*}{0.50} & \multirow[t]{12}{*}{0.25} & \multirow[t]{6}{*}{0.60} & 10 & 87 & $2222^{\dagger}$ & 165 & 728 \\
\hline & & & & $(1.000)$ & (3.172) & $(0.743)$ & $(0.696)$ \\
\hline & & & 25 & 45 & 655 & -5 & 228 \\
\hline & & & & (1.000) & $(2.826)$ & $(0.686)$ & (0.667) \\
\hline & & & 50 & 70 & 364 & 42 & 158 \\
\hline & & & & $(1.000)$ & $(2.647)$ & $(0.660)$ & $(0.650)$ \\
\hline & & \multirow[t]{6}{*}{0.80} & 10 & -155 & $1826^{\prime}$ & -53 & 637 \\
\hline & & & & (1.000) & (2.409) & $(0.405)$ & $(0.400)$ \\
\hline & & & 25 & -20 & 552 & -37 & 208 \\
\hline & & & & $(1.000)$ & (1.982) & (0.379) & $(0.374)$ \\
\hline & & & 50 & -49 & 412 & 18 & 135 \\
\hline & & & & $(1.000)$ & $(1.875)$ & $(0.365)$ & $(0.363)$ \\
\hline & \multirow[t]{12}{*}{0.33} & \multirow[t]{6}{*}{0.60} & 10 & 16 & $1613^{\dagger}$ & -23 & 671 \\
\hline & & & & (1.000) & (1.675) & (0.714) & $(0.655)$ \\
\hline & & & 25 & 49 & 597 & 24 & 274 \\
\hline & & & & (1.000) & (1.474) & (0.654) & $(\mathbf{0 . 6 3 8})$ \\
\hline & & & 50 & 14 & 377 & 33 & 163 \\
\hline & & & & (1.000) & (1.411) & $(0.626)$ & $(\mathbf{0 . 6 2 2})$ \\
\hline & & \multirow[t]{6}{*}{0.80} & 10 & 151 & $1241^{\prime}$ & -2 & 730 \\
\hline & & & & (1.000) & (1.029) & $(0.405)$ & $(0.385)$ \\
\hline & & & 25 & 36 & 461 & -13 & 262 \\
\hline & & & & $(1.000)$ & $(0.901)$ & $(0.372)$ & $(0.366)$ \\
\hline & & & 50 & -26 & 214 & -33 & 101 \\
\hline & & & & (1.000) & (0.902) & $(0.362)$ & $(\mathbf{0 . 3 5 8})$ \\
\hline
\end{tabular}


Table 2. The underlying values for $P R V_{S}(8)$ and $P R V_{R}(9)$ and their corresponding simulated values $S P R V_{S}$ (10) and $S P R V_{R}(11)$ for using the composite estimator $\hat{Y}_{C}\left(\hat{w}^{*}\right)$ with the estimated optimal weight in situations in which the coefficient of variation $C V_{x}=1 / 3,1 / 2 ; C V_{y}=1 / 4,1 / 3$; the correlation between $X_{i}$ and $Y_{i}, \rho=0.60$, 0.80; and the sample size $n=10,25$, 50. Each entry is calculated on the basis of 10,000 repeated samples.

\begin{tabular}{|c|c|c|c|c|c|c|c|}
\hline$C V_{x}$ & $C V_{y}$ & $\rho$ & $n$ & $P R V_{S}$ & $S P R V_{S}$ & $P R V_{R}$ & $S P R V_{R}$ \\
\hline \multirow[t]{12}{*}{0.33} & \multirow{6}{*}{0.25} & \multirow{3}{*}{0.60} & 10 & 0.36 & 0.27 & 0.46 & 0.43 \\
\hline & & & 25 & 0.36 & 0.33 & 0.46 & 0.44 \\
\hline & & & 50 & 0.36 & 0.34 & 0.46 & 0.46 \\
\hline & & \multirow[t]{3}{*}{0.80} & 10 & 0.65 & 0.60 & 0.45 & 0.41 \\
\hline & & & 25 & 0.65 & 0.65 & 0.45 & 0.45 \\
\hline & & & 50 & 0.65 & 0.64 & 0.45 & 0.45 \\
\hline & \multirow[t]{6}{*}{0.33} & \multirow{3}{*}{0.60} & 10 & 0.36 & 0.29 & 0.20 & 0.15 \\
\hline & & & 25 & 0.36 & 0.34 & 0.20 & 0.18 \\
\hline & & & 50 & 0.36 & 0.36 & 0.20 & 0.18 \\
\hline & & \multirow[t]{3}{*}{0.80} & 10 & 0.64 & 0.61 & 0.10 & 0.03 \\
\hline & & & 25 & 0.64 & 0.63 & 0.10 & 0.08 \\
\hline & & & 50 & 0.64 & 0.64 & 0.10 & 0.09 \\
\hline \multirow[t]{12}{*}{0.50} & \multirow[t]{6}{*}{0.25} & \multirow[t]{3}{*}{0.60} & 10 & 0.35 & 0.26 & 0.75 & 0.76 \\
\hline & & & 25 & 0.35 & 0.31 & 0.75 & 0.76 \\
\hline & & & 50 & 0.35 & 0.34 & 0.75 & 0.75 \\
\hline & & \multirow[t]{3}{*}{0.80} & 10 & 0.65 & 0.59 & 0.80 & 0.83 \\
\hline & & & 25 & 0.65 & 0.62 & 0.80 & 0.81 \\
\hline & & & 50 & 0.65 & 0.64 & 0.80 & 0.80 \\
\hline & \multirow[t]{6}{*}{0.33} & \multirow[t]{3}{*}{0.60} & 10 & 0.37 & 0.29 & 0.55 & 0.57 \\
\hline & & & 25 & 0.37 & 0.35 & 0.55 & 0.55 \\
\hline & & & 50 & 0.37 & 0.37 & 0.55 & 0.55 \\
\hline & & \multirow[t]{3}{*}{0.80} & 10 & 0.64 & 0.59 & 0.58 & 0.60 \\
\hline & & & 25 & 0.64 & 0.63 & 0.58 & 0.59 \\
\hline & & & 50 & 0.64 & 0.64 & 0.58 & 0.60 \\
\hline
\end{tabular}

respect to the MSE, although one needs to estimate $w^{*}$ from the sample data (Table 1$)$. Comparing the relative MSE of $\hat{Y}_{C}\left(\hat{w}^{*}\right)$ with that of $\hat{Y}_{C}\left(w^{*}\right)$ reveals that the percentage of inflation in the MSE because of estimation for $w^{*}$ is generally small for $n \geq 25$. For example, consider the case $C V_{x}=1 / 3, C V_{y}=1 / 3, \rho=0.60$, and $n=50$. The ratio of the MSE of $\hat{Y}_{C}\left(\hat{w}^{*}\right)$ relative to the MSE of $\hat{Y}_{C}\left(w^{*}\right)$ is 1.014 .

To study the influence due to $C V_{x}, C V_{y}$ and $\rho$, as well as the effect due to estimation of $w^{*}$ on the PRV, we summarize in Table $2 P R V_{S}(8), S P R V_{S}(10), P R V_{R}(9)$, and $S P R V_{R}$ (11) in the same set of configurations as those considered in Table 1 . When $n \geq 25$, the values of $S P R V_{S}$ agree well with those of $P R V_{S}$, and so do the values of $S P R V_{R}$ compared to $P R V_{R}$. This is consistent with the findings that the loss of precision due to estimation of the optimal weight is relatively small for $n \geq 25$ (Table 1 ). When the ratio $C V_{y} / C V_{x}$ is small $(=1 / 2)$, and $\rho$ is large $(=0.80)$, the gain in efficiency (according to $P R V_{R}$ ) for using the composite estimator as compared with the ratio estimator seems to be the largest in situations considered in Table 2.

\section{Examples}

We consider two examples, one regarding the estimation of dead fir trees via an aerial photo and the other regarding the estimation of the average sugarcane acres per county. Because the number of sampled units is small in both two examples, the bias of the ratio 
Table 3. The photo count and ground actual count of dead fir trees on ten sampled plots.

\begin{tabular}{|c|c|c|c|c|c|}
\hline \multicolumn{2}{|c}{} & $\begin{array}{c}\text { Photo } \\
\text { Count } \\
i=\end{array}$ & $\begin{array}{c}\text { Ground } \\
\text { Count } \\
X_{i}\end{array}$ & $Y_{i}$ & \multicolumn{3}{c}{$\begin{array}{c}\text { Photo } \\
\text { Count } \\
X_{i}\end{array}$} & $\begin{array}{c}\text { Ground } \\
\text { Count } \\
Y_{i}\end{array}$ \\
\hline 1 & 12 & 18 & 6 & 30 & 36 \\
2 & 30 & 42 & 7 & 12 & 14 \\
3 & 24 & 24 & 8 & 6 & 10 \\
4 & 24 & 36 & 9 & 36 & 48 \\
5 & 18 & 24 & 10 & 42 & 54 \\
\hline
\end{tabular}

Scheaffer et al. 2012, 206.

estimator can be non-negligible. However, for the purpose of illustration only, we will assume that the bias of the ratio estimator is not of our concern, as done elsewhere (Cochran 1977; Scheaffer et al. 2012).

Consider the first example regarding a forest resource manager who wishes to estimate the total number of dead fir trees in a 300 acre area of heavy infestation by use of an aerial photo. The area was divided into $(N=) 2001.5$-acre plots. We summarize in Table 3 the photo count $\left(X_{i}\right)$ and the actual ground count $\left(Y_{i}\right)$ of dead fir trees for a simple random sample of $n=10$ plots (Scheaffer et al. 2012, 206). The total number of dead fir trees obtained from the photo account is $(X=) 4200$. Given these data (Table 3), we obtain the estimated coefficient of variation $c v_{x}=0.490, c v_{y}=0.485$ and $\hat{\rho}=0.973$. These lead the optimal weight estimate to be $\hat{w}^{*}=0.037$. Because $\widehat{\operatorname{Var}}(\hat{Y})$ is much larger than $\widehat{\operatorname{Var}}\left(\hat{Y}_{R}\right)$, the estimated optimal weight $\hat{w}^{*}$ associated with the component $\hat{Y}$ of the composite estimator $\hat{Y}_{C}\left(\hat{w}^{*}\right)$ is close to 0 (or the weight $1-\hat{w}^{*}$ associated with the component $\hat{Y}_{R}$ is close to 1$)$. We get the estimates: $\hat{Y}=6120, \hat{Y}_{R}=5492$, and $\hat{Y}_{C}\left(\hat{w}^{*}\right)=5516$ with $\widehat{\operatorname{Var}}(\hat{Y})=837858, \widehat{\operatorname{Var}}\left(\hat{Y}_{R}\right)=45890$, and $\widehat{\operatorname{Var}}\left(\hat{Y}_{C}\left(\hat{w}^{*}\right)\right)=44722$. The variance estimate for $\hat{Y}_{C}\left(\hat{w}^{*}\right)$ is smaller than those of $\hat{Y}$ and $\hat{Y}_{R}$; the corresponding estimates of PRVs are $\widehat{P R V}_{S}=0.947$ and $\widehat{P R V}_{R}=0.025$.

Consider the second example regarding the estimate of the average sugarcane acres per county over $(N=) 32$ counties in Florida, Hawaii, Louisiana and Texas (United States) in 1999. We summarize in Table 4 the data on $(n=)$ the six sampled counties for years 1997 and 1999 (Scheaffer et al. 2012, 178). Given these data (Table 4), we obtain $c v_{x}=0.995$, $c v_{y}=0.930$ and $\hat{\rho}=0.993$. The optimal weight estimate is $\hat{w}^{*}=0.072$. Again, since $\widehat{\operatorname{Var}}(\hat{Y})$ is much larger than $\widehat{\operatorname{Var}}\left(\hat{Y}_{R}\right)$, the optimal weight $\hat{w}^{*}$ obtained here is also small. The average $(\bar{X}=)$ is known to be 27,752 sugarcane acres over 32 counties in 1997 . Given

Table 4. The number of sugarcane acres of the six sampled counties for year 1997 and year 1999 in the four states: Florida, Hawaii, Louisiana, and Texas.

\begin{tabular}{|l|c|c|c|c|c|c|}
\hline $\begin{array}{l}\text { County } \\
\text { State }\end{array}$ & $\begin{array}{c}\text { Hendry, } \\
\text { FL }\end{array}$ & $\begin{array}{c}\text { Kauai, } \\
\text { HI }\end{array}$ & $\begin{array}{c}\text { Saint Landry, } \\
\text { LA }\end{array}$ & $\begin{array}{c}\text { Calcasieu, } \\
\text { LA }\end{array}$ & $\begin{array}{c}\text { Iberia, } \\
\text { LA }\end{array}$ & $\begin{array}{c}\text { Cameron, } \\
\text { TX }\end{array}$ \\
\hline $\begin{array}{l}1997 \\
\text { Acreage } X_{i}\end{array}$ & 54000 & 12300 & 9100 & 1700 & 57200 & 12900 \\
\hline $\begin{array}{l}1999 \\
\text { Acreage } Y_{i}\end{array}$ & 57000 & 13900 & 15500 & 3900 & 59900 & 10400 \\
\hline
\end{tabular}

Scheaffer et al. 2012, 178 . 
these data, we obtain the estimates: $\hat{\bar{Y}}=26767, \hat{\bar{Y}}_{R}=30278$, and $\hat{\bar{Y}}_{C}\left(\hat{w}^{*}\right)=30025$ with variance estimates $\widehat{\operatorname{Var}}(\hat{\bar{Y}})=83825986, \widehat{\operatorname{Var}}\left(\hat{\bar{Y}}_{R}\right)=1595550$ and $\widehat{\operatorname{Var}}\left(\hat{\bar{Y}}_{C}\left(\hat{w}^{*}\right)\right)=1097183$. These give $\widehat{P R V}_{S}=0.987$ and $\widehat{P R V}_{R}=0.312$. Thus, we may gain a moderate amount of efficiency by use of $\hat{\bar{Y}}_{C}\left(\hat{w}^{*}\right)$ as compared with $\hat{\bar{Y}}\left(=\bar{y}_{s}\right)$ and $\hat{\bar{Y}}_{R}(=\hat{R} \bar{X})$, although the weight $1-\hat{w}^{*}=0.928$ associated with the ratio estimator in the composite estimator is large. This example may illustrate the situation in which $C V_{x}>C V_{y}$, using the composite estimator can be of use to improve the precision of the ratio estimator even for a large correlation $\rho$. We may wish to note that the estimated gain in efficiency presented in both examples does not account for the uncertainty due to estimation of the optimal weight. Thus, to further confirm this finding of gaining efficiency in the above cases with high correlation and small sample size, we have carried out additional similar simulations as described previously for correlation $\rho=0.90,0.95$. We have obtained the same results on the relative performance among different estimators with respect to the MSE as found for $\rho=0.80$ in Table 1 . For brevity, we do not present these simulation results, which are, however, available to readers upon request.

\section{Discussion}

The composite estimator $\hat{Y}\left(w^{*}\right)$ with known optimal weight can be shown to outperform the ratio estimator with respect to both bias and variance algebraically. Furthermore, the simulation results presented here suggest that both the bias and MSE of the composite estimator with the estimated optimal weight can be still smaller than those of the ratio estimator. Because there is no need for us to obtain any extra information besides the data required to apply the ratio estimator, the composite estimator proposed here should be of use to improve the efficiency of the ratio estimator in practice. We also wish to note that it is simply straightforward to extend the composite estimators discussed here to accommodate the stratified random sampling, in which the SRS is employed within each stratum.

When estimating the population mean $\bar{Y}$, Cochran (1977, 190-192) considered a class of regression estimators $\left\{\bar{y}_{s}+b_{0}\left(\bar{X}-\bar{x}_{s}\right)\right.$, where $b_{0}$ is preassigned fixed constant $\}$. It is easy to see that the composite estimator $\hat{\bar{Y}}\left(w^{*}\right)$ can be rewritten as $\bar{y}_{s}+\rho\left(C V_{y} / C V_{x}\right) \hat{R}\left(\bar{X}-\bar{x}_{s}\right)$, which is not a member of the above class of regression estimators. This is because $\rho\left(C V_{y} / C V_{x}\right) \hat{R}$ is random rather than a fixed constant. However, the composite estimator $\hat{\bar{Y}}\left(w^{*}\right)$ with the estimated optimal weight $\hat{w}^{*}$ will lead to the estimator $\bar{y}_{s}+\hat{b}_{0}\left(\bar{X}-\bar{x}_{s}\right)$, where $\hat{b}_{0}^{*}=\hat{\rho}\left(s_{y} / s_{x}\right)$ is the estimator for the constant $b_{0}^{*}\left(=\rho\left(C V_{y} / C V_{x}\right)\right)$ that minimizes the variance $\operatorname{Var}\left(\bar{y}_{s}+b_{0}\left(\bar{X}-\bar{x}_{s}\right)\right)$ under SRS (Cochran 1977, 191).

Note that we focus our attention on the classical finite-population sampling, in which the expectation is taken with respect to sampling scheme (or random permutations). As long as the auxiliary variate $X_{i}$ is highly correlated with $Y_{i}$, we may consider use of the ratio estimator accounting for the auxiliary information to improve the precision of the simple expansion estimator without making assumptions of any model relationship between $Y_{i}$ and $X_{i}$ (Cochran 1977; Scheaffer et al. 2012; Thompson 2012). Because the composite estimator includes the ratio estimator as a special case, the composite estimator with known optimal weight will have, as noted previously, the MSE smaller than the ratio estimator for all bivariate models of $\left(X_{i}, Y_{i}\right)$ under the SRS. Furthermore, we may find numerous real-life examples illustrating the use of ratio estimator under the 
SRS in data likely fitting the common linear regression model $Y_{i}=\alpha+\beta X_{i}+e_{i}$, where random errors $e_{i}$ have mean equal to 0 and constant variance $\operatorname{Var}\left(e_{i}\right)$ (Scheaffer et al. 2012, 173-186). In our simulations, we assume that the bivariate normal model for $\left(X_{i}, Y_{i}\right)$ in which all assumptions of the linear regression model with constant variance are implicitly satisfied (Casella and Berger 1990). Our simulated results (Table 1) suggest that use of composite estimators even with use of the estimated optimal weights may still substantially reduce the bias and MSE of the ratio estimator in these cases. On the other hand, the ratio estimator is often considered, provided that the population model: $Y_{i}=\beta X_{i}+e_{i}$, where random errors $e_{i}$ have mean equal to 0 and variance $\operatorname{Var}\left(e_{i}\right)$ proportional to $X_{i}$ is assumed under the super-population model-based approach (SPMBA) (Royall 1970). This is because the ratio estimator $\hat{Y}_{R}$ is the best linear unbiased estimator for the population total $Y$ with respect to random errors assumed in this linear model under the SPMBA (Cochran 1977, 158-159). However, for a given correlation $\rho$ between $X_{i}$ and $Y_{i}$, creating a bivariate model for $\left(X_{i}, Y_{i}\right)$ with the conditional mean $E\left(Y_{i} \mid X_{i}\right)=\beta X_{i}$ and conditional variance $\operatorname{Var}\left(Y_{i} \mid X_{i}\right)=\sigma^{2} X_{i}$ is extremely difficult. Thus, a topic for future research is to extend our simulation results under the SRS for a given fixed correlation $\rho$ to cover cases with the conditional variance $\operatorname{Var}\left(Y_{i} \mid X_{i}\right)$ proportional to $X_{i}$ when one needs to estimate the optimal weight for the composite estimator from data.

Finally, when $X_{i}$ and $Y_{i}$ are dichotomous (i.e., $X_{i}$ and $Y_{i}=1$ if subject $i$ possesses the characteristics of interest, and $=0$, otherwise), we note that one may follow the same arguments as presented here and derive the optimal weight minimizing the variance (or MSE) of the composite estimator under the SRS. We can further derive the PRVs for using the composite estimator as compared with the ratio and simple expansion estimators. We leave some details in Appendix 2 (Subsection 7.2.) for readers' information.

\section{Appendix}

\subsection{Appendix 1}

Note that

$$
\begin{aligned}
E(\hat{Y}-Y)\left(\hat{Y}_{R}-Y\right) & =E(\hat{Y}-Y)\left(\hat{Y}_{R}-E\left(\hat{Y}_{R}\right)+E\left(\hat{Y}_{R}\right)-Y\right) \\
& =E(\hat{Y}-Y)\left(\hat{Y}_{R}-E\left(\hat{Y}_{R}\right)\right)+E(\hat{Y}-Y)\left(E\left(\hat{Y}_{R}\right)-Y\right) .
\end{aligned}
$$

Because $\left(E\left(\hat{Y}_{R}\right)-Y\right)$ is constant and $E(\hat{Y})=Y$, the second part in (A.1) is zero. Therefore, we have $\operatorname{Cov}\left(\hat{Y}, \hat{Y}_{R}\right)=E(\hat{Y}-Y)\left(\hat{Y}_{R}-Y\right)$.

Furthermore, note that

$$
\begin{aligned}
E(\hat{Y}-Y)\left(\hat{Y}_{R}-Y\right) & =E\left(N \bar{y}_{s}-Y\right)(\hat{R} X-Y)=N^{2} \bar{X} E\left(\bar{y}_{s}-\bar{Y}\right)\left(\left(\bar{y}_{s}-R \bar{x}_{s}\right) / \bar{x}_{s}\right) \\
& \approx N^{2} E\left(\bar{y}_{s}-\bar{Y}\right)\left(\left(\bar{y}_{s}-\bar{Y}-R\left(\bar{x}_{s}-\bar{X}\right)\right)\right. \\
& =N^{2}\left[E\left(\bar{y}_{s}-\bar{Y}\right)^{2}-R E\left(\bar{y}_{s}-\bar{Y}\right)\left(\bar{x}_{s}-\bar{X}\right)\right] \\
& =N^{2}(1-f)\left[S_{y}^{2}-R S_{x y}\right] / n \\
& =N^{2}(1-f) \bar{Y}^{2}\left[C V_{y}^{2}-\rho C V_{x} C V_{y}\right] / n
\end{aligned}
$$


Note also that

$$
\begin{aligned}
E\left(\hat{Y}_{R}-Y\right)^{2} & =N^{2} \bar{X}^{2} E(\hat{R}-R)^{2}=N^{2} \bar{X}^{2} E\left(\left(\bar{y}_{s}-R \bar{x}_{s}\right) / \bar{x}_{s}\right)^{2} \\
& \approx N^{2} E\left(\bar{y}_{s}-R \bar{x}_{s}\right)^{2}=N^{2} E\left(\bar{y}_{s}-\bar{Y}-R\left(\bar{x}_{s}-\bar{X}\right)\right)^{2} \\
& =N^{2}\left[E\left(\bar{y}_{s}-\bar{Y}\right)^{2}+R^{2}\left(\bar{x}_{s}-\bar{X}\right)^{2}-2 R E\left(\bar{y}_{s}-\bar{Y}\right)\left(\bar{x}_{s}-\bar{X}\right)\right] \\
& =N^{2}(1-f)\left[S_{y}^{2}+R^{2} S_{x}^{2}-2 R S_{x y}\right] / n \\
& =N^{2}(1-f) \bar{Y}^{2}\left[C V_{y}^{2}+C V_{x}^{2}-2 \rho C V_{x} C V_{y}\right] / n
\end{aligned}
$$

Because $E\left(\hat{Y}_{R}\right)$ is actually not equal to $Y$, it can be more appropriate to call $E\left(\hat{Y}_{R}-Y\right)^{2}$ (A.3) the MSE of $\hat{Y}_{R}$ as what was done by Sukhatme et al. (1984, 192). However, the relative bias $E\left(\hat{Y}_{R}-Y\right) / Y \approx(1-f) C V_{x}^{2}\left(1-\rho C V_{y} / C V_{x}\right) / n$ converges to 0 as $n$ increases. Therefore, despite of $E\left(\hat{Y}_{R}\right) \neq Y$, Formula (A.3) is also the traditional variance formula used for $\operatorname{Var}\left(\hat{Y}_{R}\right)$ in many textbooks (Cochran 1977; Scheaffer et al. 2012; Govindarajulu 1999; Thompson 2012).

Because $E\left(\hat{Y}_{R}-Y\right)^{2} \approx \operatorname{Var}\left(\hat{Y}_{R}\right)$ and $\operatorname{Cov}\left(\hat{Y}, \hat{Y}_{R}\right)=E(\hat{Y}-Y)\left(\hat{Y}_{R}-Y\right)$, the optimal weight $w^{*}$ minimizing the variance $\operatorname{Var}\left(\hat{Y}_{C}(w)\right)$ of the composite estimator $\hat{Y}_{C}(w)=$ $w \hat{Y}+(1-w) \hat{Y}_{R}$ is equivalent to minimizing the MSE $E\left(\hat{Y}_{C}(w)-Y\right)^{2}$. To derive the optimal weight $w^{*}$ minimizing $\operatorname{Var}\left(\hat{Y}_{C}(w)\right)$, we set the first derivative of $\operatorname{Var}\left(\hat{Y}_{C}(w)\right)$ taken with respect to $w$ equal 0 and solve the equation.

\subsection{Appendix 2}

When $X_{i}\left(\right.$ and $\left.Y_{i}\right)=1$ if subject $i$ possesses the characteristics of interest, and $=0$, otherwise. One can show that $C V_{x}=\sqrt{N\left(1-P_{x}\right) /\left[(N-1) P_{x}\right]}$, where $P_{x}=\sum_{i=1}^{N} X_{i} / N$ represents the proportion of subjects possessing the characteristics of interest in the population of $X_{i}$. Similarly, $C V_{y}=\sqrt{N\left(1-P_{y}\right) /\left[(N-1) P_{y}\right]}$, where $P_{y}=\sum_{i=1}^{N} Y_{i} / N$ represents the proportion of subjects possessing the characteristics of interest in the population of $Y_{i}$. Note that $\rho$ is simply identical to the phi $\varphi$ correlation for dichotomous data defined elsewhere (Fleiss et al. 99). Thus, the optimal weight $w$ for the composite estimator $\hat{Y}_{C}(w)=w\left(N \hat{p}_{y}\right)+(1-w)\left(\hat{p}_{y} / \hat{p}_{x}\right)\left(N P_{x}\right) \quad\left(\right.$ where $\hat{p}_{y}=\sum_{i \in c} y_{i} / n$ and $\hat{p}_{x}=$ $\left.\sum_{i \in c} x_{i} / n\right)$ minimizing variance $\operatorname{Var}\left(\hat{Y}_{C}(w)\right)$ is

$$
w^{*}=1-\varphi \sqrt{O R_{x y}}
$$

where $O R_{x y}=P_{x}\left(1-P_{y}\right) /\left[\left(1-P_{x}\right) P_{y}\right]$ is the odds ratio $(\mathrm{OR})$ of the marginal proportions of possessing the characteristics between populations of $X_{i}$ and of $Y_{i}$. Note that this $O R_{x y}$ is different from the OR, $\left(p_{11} p_{00}\right) /\left(p_{10} p_{01}\right)$, where $p_{r s}=P\left(X_{i}=r, Y_{i}=s\right), r=1,0$ and $s=1,0$. The latter OR is related to the $\varphi$ correlation, measuring the strength of association between $X_{i}$ and $Y_{i}$. Note that the OR has many mathematically inherent and desirable properties and is one of the most common-used indices measuring the extent of association between variables in Epidemiology (Lui 2004, 89-90). On the basis of Formula (A.4), we see that the higher the $\varphi$ or the larger the $O R_{x y}$, the smaller is the optimal weight $w^{*}$ 
(subject to $w^{*} \geq 0$ ). The PRV based on (8) by use of $\hat{Y}_{C}\left(w^{*}\right)$ instead of $\hat{Y}$ becomes

$$
P R V_{S}=\varphi^{2}
$$

Furthermore, the PRV based on (9) by use of $\hat{Y}_{C}\left(w^{*}\right)$ instead of $\hat{Y}_{R}$ is

$$
P R V_{R}=\left(1-\varphi \sqrt{O R_{x y}}\right)^{2} /\left[\left(1-\varphi \sqrt{O R_{x y}}\right)^{2}+O R_{x y}\left(1-\varphi^{2}\right)\right]
$$

When the prevalence rate of possessing the characteristics of interest between populations $X_{i}$ and $Y_{i}$ are equal (i.e., $\left.O R_{x y}=1\right), P R V_{R}$ Formula (A.6) simplifies to $(1-\varphi) / 2$. A systematic comparison of the performance for the composite estimator with the estimated optimal weight minimizing the variance (or the MSE) versus its two component estimators in dichotomous data can be a small research project.

\section{References}

Casella, G. and R.L. Berger. 1990. Statistical Inference. Belmont, CA: Duxbury.

Cochran, W.G. 1977. Sampling Techniques (3rd ed.). New York: Wiley.

Fleiss, J.L., B. Levin, and M.C. Paik. 2003. Statistical Methods for Rates and Proportions (3rd ed.). New York: Wiley. DOI: https://doi.org/10.1002/0471445428.

Govindarajulu, Z. 1999. Elements of Sampling Theory and Methods. Upper Saddle River, NJ: Prentice Hall.

Lee, S.E., P.R. Lee, and K-II. Shin. 2016. "A Composite Estimator for Stratified TwoStage Cluster Sampling." Communications for Statistical Applications and Methods 23: 47-55. DOI: https://doi.org/10.5351/CSAM.2016.23.1.047.

Lui, K.-J. and W.G. Cumberland. 1991. "A Model-Based Approach: Composite Estimators for Small Area Estimation.” Journal of Official Statistics 7: 69-76.

Lui, K.-J. 2004. Statistical Estimation of Epidemiological Risk. New York: Wiley. DOI: https://doi.org/10.1002/0470094087.

Pascual, J.N. 1961. "Unbiased Ratio Estimators in Stratified Sampling." Journal of American Statistical Association 56: 70-87. DOI: https://doi.org/10.2307/2282332.

Royall, R.M. 1970. "On Finite Population Sampling Theory under Certain Linear Regression Models.” Biometrika 57: 377-387. DOI: https://doi.org/10.1093/biomet/ 57.2.377.

Sahoo, L.N. 1987. "On a Class of Almost Unbiased Estimators for Population Ratio." Statistics 18: 119-121. DOI: https://doi.org/10.1080/02331888708801998.

SAS Institute Inc. 2009. SAS/STAT 9.2 User's Guide (2nd ed.). Cary, NC: SAS Institute. Schaible, W.L. 1978. "Choosing Weight for Composite Estimators for Small Area Statistics." In Proceedings of the Section on Survey Research Methods, 741-746. Washington: American Statistical Association.

Schaible, W.L. 1979. "A Composite Estimator for Small Area Statistics.” In Proceedings of the Section on Social Statistics, 1017-1021. Washington: American Statistical Association

Scheaffer, R.L., W. Mendenhall III, R.L. Ott, and K.G. Gerow. 2012. Elementary Survey Sampling (7th ed.). Boston, MA: Brooks/Cole. 
Sukhatme, P.V., B.V. Sukhatme, S. Sukhatme, and C. Asok. 1984. Sampling Theory of Surveys Applications (3rd ed.). Ames, Iowa: Iowa State University Press.

Thompson, S.K. 2012. Sampling (3rd ed.). New York: Wiley. DOI: https://doi.org/ $10.1002 / 9781118162934$.

Received November 2018

Revised April 2019

Accepted November 2019 